We agree that there is no evidence to support the use of dexamethasone in meningococcal septicaemia. As previously highlighted, we did not find any evidence of benefit when it was used in meningococcal meningitis either. We reiterate that further clinical studies are urgently required to clarify these important issues.

CS NANAYAKKARA Consultant paediatrician

$\mathrm{R} \operatorname{COX}$

Paediatric Directorate,

Consultant microbiologist

Kettering General Hospital NHS Trust,

Kettering,

Northamptonshire NN16 8UZ

1 Calman KC. Meningococcal infection: meningitis and septicaemia London: Department of Health, 1994. (PL CMO (94)2.) 2 Control of meningococcal disease. Communicable Disease Report 1993;3:229-30.

\section{Risk factors for breast cancer}

\section{Socioeconomic differences might be explained by body mass}

EDITOR,-Andrew G Carnon and colleagues could not explain socioeconomic differences in survival from breast cancer by difference in tumour stage or biology. ${ }^{1}$ An alternative explanation might lie in differences in body mass. Heavier women have a worse prognosis from breast cancer than lighter women. ${ }^{2}$ Also, heavier women are less likely to attend screening programmes than lighter women. ${ }^{3}$ Therefore heavy women may be in double jeopardy of dying of breast cancer: they are less likely to attend for screening, so cancer is diagnosed later and when it is diagnosed the prognosis is poor. Given that social status is inversely correlated with body weight, ${ }^{4}$ differences in survival among social groups after breast cancer has been diagnosed may be attributable simply to class differences in body mass. Furthermore, the phenomenon whereby women who do not attend breast screening programmes have a higher mortality from the disease than those who do attend, ${ }^{5}$ even though the incidence of disease is similar, may again be explained by the increased body mass of those who do not attend relative to those who do.

\section{DAVID TORGERSON} Research fellow

Health Economics and Health Services Research Units,

University of Aberdeen,

Aberdeen AB9 2ZD

1 Carnon AG, Ssemwogerere A, Lamont DW, Hole DJ, Mallon $\mathrm{EA}$, George WD, et al. Relation between socioeconomic deprivation and pathological prognostic factors in women with breast cancer. $B M Y$ 1994;309:1054-7. (22 October.)

2 Bastarrachea J, Hortobagyi GN, Smith TL, Shu-Wan C, Buzdar $\mathrm{AU}$. Obesity as an adverse prognostic factor for patients receiving adjuvant chemotherapy for breast cancer. Ann Inter Med 1994;119:18-25.

3 Torgerson DJ, Donaldson C, Garton MJ, Russell IT, Westland M, Reid DM. Population screening for osteoporosis: do nonattenders have a lower risk of osteoporosis? Osteoporosis International 1994;4:149-53.

4 West R. Obesity. London: Office of Health Economics, 1994.

5 UK Trial of Early Detection of Breast Cancer Group. First results on mortality reduction in the UK trial of early detection of on mortality reduction in the UK
breast cancer. Lancet 1988;ii:411-6.

\section{Pollutants and pesticides may be important}

EDrToR,-K McPherson and colleagues fail to mention xeno-oestrogens and progesterone deficiency as important risk factors for breast cancer. ${ }^{1}$ Recently, several pollutants and pesticides have been recognised as having potent oestrogenic effects in the body, and these are likely to be a contributing factor in the development of breast cancer. ${ }^{2}$ Dicophane (DDT), which is stored in the body's fatty tissue as dichlorodiphenyldichloroethylene (DDE), and polychlorinated phenols are two such pesticides.
Fuchs has reviewed some of the evidence linking pesticides and breast cancer. ${ }^{3}$ Dr Mary Wolff, of the Mount Sinai School of Medicine, measured blood concentrations of DDE in women and found that women with high concentrations were four times as likely to have breast cancer as those with lower concentrations. Another study, published in the Archives of Environmental Health in 1993, found that patients with breast cancer had more than twice the concentration of polychlorinated phenols and DDE in their breast tissue as comparable women without cancer. Mortality from breast cancer declined in Israel after dicophane and other carcinogenic pesticides, which occurred in high concentrations in milk and dairy products, were banned in 1978 .

There is strong evidence that "oestrogen dominance" is a major risk factor for breast cancer and that progesterone has a protective effect. ${ }^{4}$ In a prospective study by Cowan et al premenopausal women with low progesterone concentrations were found to have 5.4 times the risk of developing premenopausal breast cancer of those with normal concentrations and to have a 10 -fold increase in deaths from all malignant neoplasms. There is a good case for measuring at least serum progesterone concentrations in women at high risk and in those with breast cancer and considering progesterone supplementation if concentrations are low. Synthetic progestogens have very different effects on the body from progesterone itself. Natural progesterone (derived from the Mexican yam and available commercially) can be conveniently given as a transdermal cream or sublingual drops and is the compound of choice in restoring hormone concentrations in such women.

PETER J LEWIS Complementary physician

Centre for the Study of Complementary Medicine,

Southampton,

Hampshire SO15 2DT

1 McPherson K, Steel CM, Dixon JM. Breast cancer-epidemiology, risk factors, and genetics. $B M \mathcal{F}^{1}$ 1994;309:1003-6. (15 October.)

2 Raloff J. Ecocancers: do environmental factors underlie a breast cancer epidemic? Science News 1993;144:10-3.

3 Fuchs $\mathrm{K}$. The pesticide and breast cancer connection. Women's Health Letter $1994 \mathrm{Jan}$

4 Lee JR. Natural progesterone. Sebastopol, CA: BLL Publishing, 1993.

5 Cowan LD, Gordis L, Tonascia JA, Jones GS. Breast cancer incidence in women with progesterone deficiency. $A m$ Epidemiol 1981;114:209-17.

\section{Mammography after treatment for breast cancer}

EDITOR,-Eva Grunfeld and colleagues believe that they may have identified a widespread problem relating to the follow up of patients with breast cancer. ${ }^{1}$ In fact, all they have identified is the inadequacy of a survey by non-clinicians analysing a single factor identified in a patient's notes without taking heed of clinical features.

In most large breast units mammography is included in the follow up protocol for patients who have received treatment for breast cancer. On many occasions, however, mammography that may have been planned sometime in advance becomes inappropriate owing to a change in clinical circumstances, including the development of cytologically or histologically proved recurrent disease, distant metastases, or unrelated coexisting medical problems. On occasions mammography is delayed because of continued induration of the breast after surgery and radiotherapy. In addition, patients may be disinclined to undergo further mammography. Accordingly, Grunfeld and colleagues' concern that "over a third of patients did not undergo mammography at the expected time" is unfounded without further analysis of the reasons for the delay. A clinical protocol does not fail because clinical circumstances may preclude adherence to it.

Breast Clinic,

Northampton General Hospital NHS Trust,

Northampton NN1 5BD

1 Grunfeld E, Mant D, Vessey MP, Cole DJ, Stewart JA. Mammography after treatment for breast cancer. $B M \mathcal{F} 1994$; 309:665-6. (10 September.)

\section{Author's reply}

EDITOR,-S J A Powis is concerned that we did not consider clinical circumstances in our definition of delay. This is not correct. All the medical records in the 145 cases were reviewed by a clinician, and the review took into account the complete clinical picture according to the medical records. When a delay in mammography was found no clinical circumstances that could explain why it had occurred was evident on this review. None of the patients had recurrent disease or distant metastases, which Powis cites as possible reasons for not performing mammography. In addition, almost all of these patients had had mammography at least once after completing primary treatment, making it unlikely that mammography was subsequently postponed until the resolution of induration from surgery or radiotherapy.

It is possible that in a small proportion of patients the delay was due to a coexisting medical problem or the patient's choice. To allow for these factors we reported delays of six and 12 months. This allowed clinicians reading the report to decide which interval they thought was clinically important.

We clearly stated the limitations of our findings -that they were restricted to two hospitals and 145 patients-and that we cannot say for certain that we have identified a more widespread problem. We also clearly stated that the benefit and optimum frequency of mammographic surveillance remain uncertain and should be the subject of a randomised trial (we recognise the methodological difficulties of such a trial). We are concerned, however, that it may be neither a clinical decision nor the patient's choice that decides whether a policy of mammographic surveillance adopted by a clinician gets implemented. Rather, it may be the unfortunate consequence of breast cancer clinics being overburdened.

EVA GRUNFELD

Department of Medicine, University of Ottawa, Assistant profess Ottawa, Ontario, Canada

\section{Medical staffing in general hospitals}

EDITOR,-I support E R Williams and colleagues' proposal for a core unit in hospitals 1 -a model similar to the facilitating unit ${ }^{2}$ - but believe that this alone will not be sufficient in the future. Other innovations need consideration in addition.

Firstly, the respective roles of general practitioners and hospital specialists must be clarified. The NHS is unique in having a primary care infrastructure, but we do not seem to utilise its potential fully. Thus, given that general practitioners are the guardians of patients' health and have the relevant histories, why do junior doctors spend time clerking patients and why cannot such histories be shared by means of the existing information technology?

Secondly, we need to review the place of traditional hospital specialties. Why do we continue to have separate medical and surgical gastroenterology services or rheumatology and orthopaedic services, for example?

Overall we need to decide what care should be 